\title{
Mars Orbiter magnetometer of China's First Mars Mission Tianwen-1
}

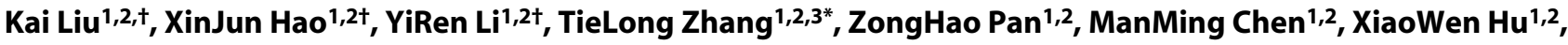 \\ Xin $\mathrm{Li}^{1,2}$, ChengLong Shen ${ }^{1,2}$, and YuMing Wang ${ }^{1,2^{*}}$ \\ ${ }^{1}$ Chinese Academy of Sciences Key Laboratory of Geospace Environment, School of Earth and Space Sciences, University of Science and Technology of China, \\ Hefei 230026, China; \\ ${ }^{2}$ Chinese Academy of Sciences Center for Excellence in Comparative Planetology, University of Science and Technology of China, Hefei 230026, China; \\ ${ }^{3}$ Space Research Institute, Austrian Academy of Sciences, Graz, Austria
}

Citation: Liu, K., Hao, X. J., Li, Y. R., Zhang, T. L., Pan, Z. H., Chen, M. M., Hu, X. W., Li, X., Shen, C. L., and Wang, Y. M. (2020). Mars Orbiter magnetometer of China's First Mars Mission Tianwen-1. Earth Planet. Phys., 4(4), 384-389. http://doi.org/10.26464/epp2020058

\begin{abstract}
As one of the seven scientific payloads on board the Tianwen-1 orbiter, the Mars Orbiter Magnetometer (MOMAG) will measure the magnetic fields of and surrounding Mars to study its space environment and the interaction with the solar wind. The instrument consists of two identical triaxial fluxgate magnetometer sensors, mounted on a 3.19 meter-long boom with a seperation of about $90 \mathrm{~cm}$. The dual-magnetometers configuration will help eliminate the magnetic field interference generated by the spacecraft platform and payloads. The sensors are controlled by an electric box mounted inside the orbiter. Each magnetometer measures the ambient vector magnetic field over a wide dynamic range (to 10,000 nT per axis) with a resolution of $1.19 \mathrm{pT}$. Both magnetometers sample the ambient magnetic field at an intrinsic frequency of $128 \mathrm{~Hz}$, but will operate in a model with alternating frequency between 1 and $32 \mathrm{~Hz}$ to meet telemetry allocations.
\end{abstract}

Keywords: Mars; magnetic field; magnetometer; Tianwen-1; spaceflight instrumentation

\section{Introduction}

\subsection{Martian Magnetic Fields}

Understanding the magnetic field environment around Mars is one of the primary scientific targets of the Tianwen-1 mission. Unlike the Earth, Mars has no global magnetic field, which has been confirmed by observations from the Mars Global Surveyor (MGS) mission (Acuña et al., 1998); therefore, it has no dynamo at present. The interaction with the solar wind forms a magnetosphere surrounding Mars (Figure 1).

The solar wind, a supersonic stream of plasma originating from the Sun, carries with it the interplanetary magnetic field (IMF). Because magnetic field lines cannot pass through an obstacle (such as Mars), the expanding solar wind drags the frozen-in IMF around the planet and forms a magnetosphere (Brain, 2006). The magnetosphere of Mars is a multitiered interaction region. The upstream outer boundary is the bow shock. The solar wind transitions from supersonic to subsonic as it crosses the bow shock into the hotter, denser, more turbulent magnetosheath (Espley et al., 2004). The lower boundary of the magnetosheath is the magnetic pileup boundary (MPB; Bertucci et al., 2003). Below the MPB

'Equally Contribution.

Correspondence to: T. L. Zhang, tlzhang@ustc.edu.cn

Y. M. Wang, ymwang@ustc.edu.cn

Received 11 JUL 2020; Accepted 29 JUL 2020.

Accepted article online 03 AUG 2020.

(C) 2020 by Earth and Planetary Physics. is the ionosphere or photoelectron boundary (PEB), which marks the narrow transition between plasma dominated by ions of solar wind origin and plasma dominated by ions of planetary origin (Connerney et al., 2015). The magnetic field extends well downstream and forms the magnetotail on the night side.

Measurements from the MGS magnetometer revealed the existence of powerful magnetic crustal fields on the planet's surface, especially in the southern highlands, which are far more powerful than those found on Earth (Acuña et al., 1998). The intensely magnetized Martian crust can establish order over scale lengths of hundreds of kilometers, which can perturb the global interaction at low altitudes, dramatically altering the nature of the interaction with the solar wind (Dong CF et al., 2014). The presence of these crustal fields gives rise to local mini-magnetospheres, small areas where the lines of the magnetic field locally protect the planet surface from electrically charged particles. Numerical simulations have amply demonstrated that the strong magnetic fields associated with the southern highlands have a shielding effect that reduces the ion escape flux (Ma YJ et al., 2004; Dong CF et al., 2014).

\subsection{Scientific Objectives of MOMAG}

During the Tianwen-1 mission, Orbiter will repeatedly cross the boundaries of the Martian magnetosphere, such as the bow shock, MPB, and PEB. On board the Orbiter, the Mars Orbiter Magnetometer (MOMAG) will measure the ambient magnetic field in the near-Mars space, from the solar wind to the magnetosheath and ionosphere. The magnetic fields in different regions have different characteristics, and systematic changes indicate their 


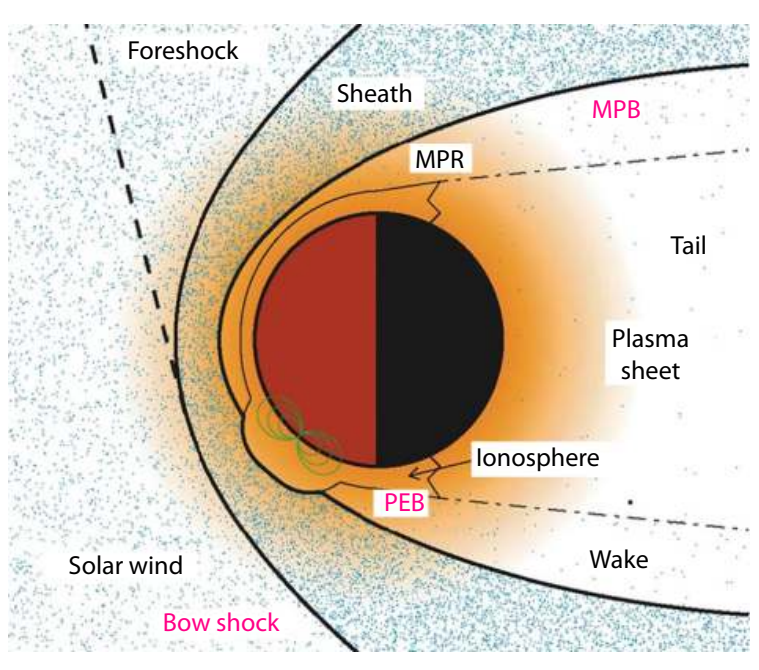

Figure 1. Diagram of the global Martian solar wind interaction (Brain, 2006). Orange shading indicates the density of planetary neutrals. Blue indicates the relative density of solar wind ions in different plasma regions (labeled in black), separated by different plasma boundaries (labeled in magenta). MPR, magnetic pileup region; MPB, magnetic pileup boundary; PEB, photoelectron boundary.

boundaries. Thus, clear definitions of these boundaries will be derived from the number of magnetic field observations.

The rover of the Tianwen-1 mission also carries a magnetometer, called the Mars Rover Magnetometer (RoMAG). When Orbiter nears its lowest altitude, MOMAG and RoMAG will make joint observations of the Martian magnetic field from the surface to an altitude of hundreds of kilometers. These joint observations will help us understand the origin and evolution of the Martian ionosphere. Both MOMAG and RoMAG could potentially be operating in the same strong crustal field; therefore, the local mini-magnetosphere and its influences on the global interaction will be studied.

The Mars Ion and Neutral Particle Analyzer (MINPA) and the Mars Energetic Particle Analyzer (MEPA), both on board Orbiter, will measure the particles and mass spectrometry in situ. Together with MOMAG, a plenthy of space environment observations will be obtained to advance our understanding of the formation and evolution of the Mars magnetosphere and its complex interaction with the solar wind, which may further provide us clues about the habitable evolution of Mars.

\section{Instrument Design}

\subsection{Overview}

Fluxgate magnetometers (FGM) are the most widely used magnetometers for space applications. MOMAG configuration consists of two identical FGMs. The FGM comprises a vector-compensated ring-core sensor and field-programmable gate array (FPGA)-based digital signal processing electronics, and it has the flight heritage from magnetometers aboard the Rosetta Lander Philae (Auster et al., 2007), Venus Express (Zhang TL et al., 2006), and THEMIS (Auster et al., 2008).

The measurement principle is based on a soft-magnetic ring core being driven into saturation by an AC excitation current. An ex- ternal magnetic field will distort the symmetry of the magnetic flux within the core, and thus influence the even harmonics of the excitation frequency. The sensors and electronics are designed for selective measurement of the second harmonic of the excitation frequency. A feedback current is generated to compensate for the external field at the ring-core position by a Helmholtz coil system. The component values of the external field are calculated by using the settings of the field-proportional feedback current and the remaining field at the core position.

Both sensors are connected to the respective electronics with a sensor harness of several meters. The two sensors on the same boom form a geometry for gradiometer measurements along the boom. One FGM sensor is located at the outboard (OB) end of the boom and the other is mounted inboard (IB) at 0.9 meter from the $\mathrm{OB}$. The total boom length from the interface to tip is 3.19 meters, as shown in Figure 2.

MOMAG instrument functions as a dual magnetometer to separate the magnetic field disturbance generated by the spacecraft from the ambient field, which is required for data correction and calibration purposes (Ness et al., 1971; Hedgecock, 1975; Neubauer, 1975; Georgescu et al., 2008). The total weight of the sensors plus electronics is $2.769 \mathrm{~kg}$, and the power required is 3.81 W (Table 1).

\subsection{Sensors}

Fluxgate sensors in general consist of a set of coil systems with a highly permeable ferromagnetic core, which often use a ring core (Primdahl, 1979). The ring cores used for MOMAG have been developed by Karl Heinz Fornaçon in Germany for more than 30 years (Müller et al., 1998). Two orthogonal intertwined ring cores of different sizes are located in the center of the sensor. The smaller ring core measures the magnetic field in the $X$ and $Z$ directions, whereas the larger one is used for the $Y$ and $Z$ directions (see Figure 3a).

The ring cores are integrated into two three-dimensional coil systems (Figure 3b). The inner coil system is used for picking up the second harmonics of the driving frequency, at which the signal amplitude is proportional to the external magnetic field. The outer coil system is constructed in a Helmholtz configuration and is used as a compensation system to cancel out the field at the ring-

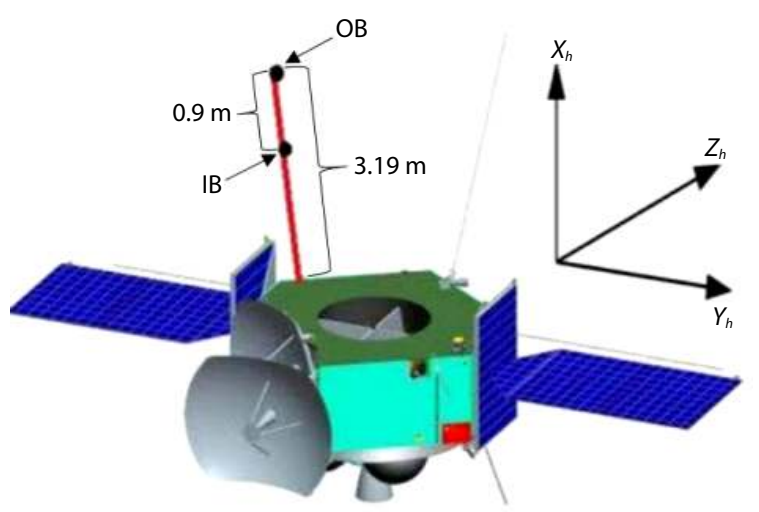

Figure 2. Illustration of MOMAG on board Tianwen-1 Orbiter. OB, outboard; IB, inboard. 

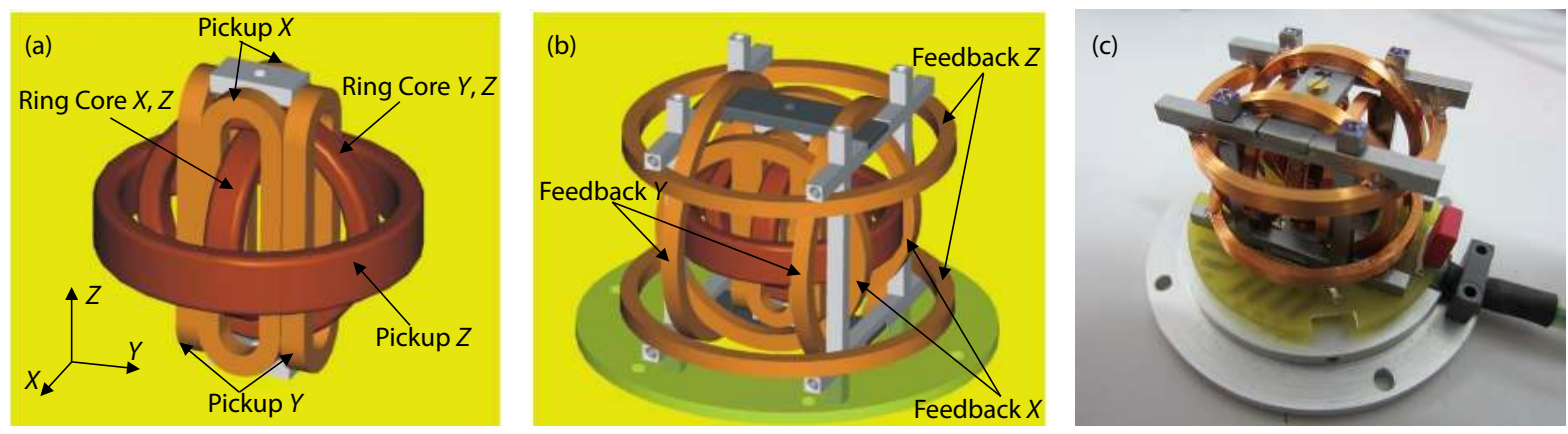

Figure 3. (a, b) Fluxgate magnetometer (FGM) sensor of the 3-D model (Auster et al., 2008). (c) Photograph of an FGM sensor.

core position. The pickup coil system is located at the closest possible position to the ring cores to enhance the signal-to-noise ratio. The feedback system is made of only copper and aluminum. Both materials feature a similar thermal expansion coefficient of about 20 ppm, which guarantees a wide temperature measurement range and a temperature-independent axis alignment.

\subsection{Electronics}

The electronics box (EBOX, Figure 4) of the magnetometer consists of three printed circuit boards: the inboard sensor electronics (IBE), the outboard sensor electronics (OBE), and the instrument control unit (ICU). Each board is fixed in an independent frame fabricated from magnesium alloy type MB15, and the boards are then stacked one on another. The three electronics boards are connected by two box-external interconnection harnesses with sub-miniature-D and micro-miniature connectors.

The sensor electronics consist of excitation circuitry, preamplifiers, analog-to-digital (AD) converters (ADCs), and digital-to-analog (DA) converters (DACs) with a trailing feedback current source and a processing unit implemented on an FPGA. The sensor electronics (Figure 5) generate an excitation AC current (fundamental frequency of $\sim 9.6 \mathrm{kHz}$ ) to drive the soft-magnetic core material deep into positive and negative saturation. According to the principle of FGM operation, the external magnetic field distorts the symmetry of the magnetic flux and generates field-proportional even harmonics of the drive frequency in the sense coils. The induced voltage in the sense coil is digitized immediately after the preamplifier at four times the excitation frequency. The "frontend" signal processing (synchronous detection and integration, and calculation of the feedback signals) is performed by logic blocks within an Actel A3P1000 FPGA. To increase the overall linearity and stability of the magnetometer, a feedback field is ap-

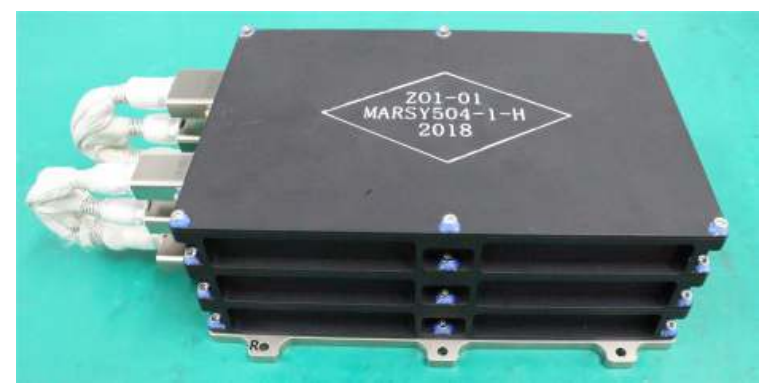

Figure 4. The electronics box (EBOX) of MOMAG. plied to the sensor through a set of Helmholtz feedback coils. For this purpose, two cascaded 16-bit DACs $\left(D^{2} C_{f}\right.$ and $\left.D A C_{c}\right)$, forming an 18-bit DAC, and a voltage-controlled current source integrated in a hybrid integrated circuit (IC) are used. An FPGA internal RISC (reduced instruction set computing) processor calculates the magnetic field value from the accumulated $A D C$ values and the last set of DAC feedback values with the equation $B=K_{1} \times A D C+$ $K_{2} \times D A C_{f}+K_{3} \times D A C_{c}$ and transmits the results to the ICU for further processing. The dynamic range is $\pm 10,000 \mathrm{nT}$ with a resolution of 1.19 pT (Table 1).

The ICU consists mainly of short-circuit protection circuitry, a power relay, soft-start circuitry, the housekeeping ADC, enable/inhibit control circuitry, and an FPGA (Figure 6). The basic tasks of the ICU include the following:

- Sensor electronics communication: scientific and housekeeping data receiving, SLIP (Serial Line Internet Protocol) decoding and encoding, sending commands for firmware selection and parameter uploading, exception handling, RS-422 interface servicing.

- Payload controller communication: telecommand reception, decoding and acknowledging, scientific and housekeeping data transmission, exception handling, RS-422 interface servicing.

- Global time synchronization.

- Synchronization of the magnetic field measurements of IBE and OBE.

- Data filtering and compression via seven stages of the 17th-order finite impulse response (FIR) half-band filters implemented in the FPGA. Two sets of coefficients are implemented inside the FP$\mathrm{GA}$, and these coefficients can be changed by telecommand.

- Scientific data packet and housekeeping data packet generation.

- Online monitoring of the currents of the secondary power supplies of the IBE and OBE. Monitoring is used to protect the electronic parts from the single-event latch. When one or more currents exceed the thresholds for $30 \mathrm{~s}$, the sensor electronics will be powered off by the enable/inhibit control circuitry.

\section{Calibration}

\subsection{Overview}

Fluxgate magnetometers do not perform absolute measurements, and they need to undergo ground and in-flight calibration 


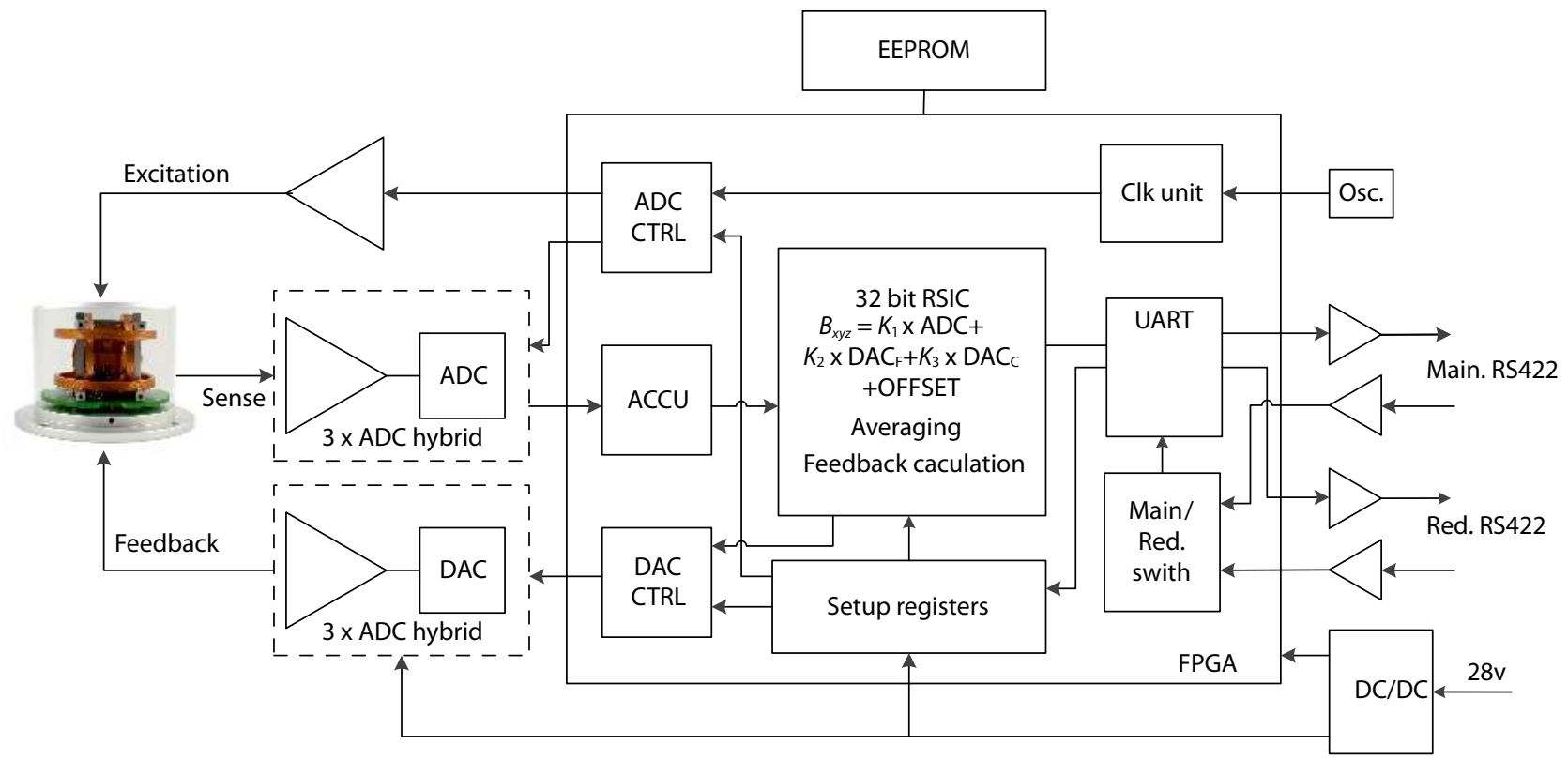

Figure 5. Block diagram of the Mars Orbiter Magnetometer (MOMAG) sensor electronics. ADC, analog-to-digital (AD) converter; $D A C$, digital-toanalog (DA) converter; ACCU, accumulator; ADC CTRL, AD converter control; DAC CTRL, DA converter control; EEPROM, electrically erasable programmable read-only memory; RSIC, reduced instruction set computing; Clk, clock; UART, universal asynchronous receiver/transmitter; FPGA, field-programmable gate array.

Table 1. Technical specifications of MOMAG.

\begin{tabular}{lll}
\hline \multicolumn{1}{c}{ Parameter } & \multicolumn{1}{c}{ Unit } & \multicolumn{1}{c}{ Value } \\
\hline Mass & Inboard sensor & $0.572 \mathrm{~kg}$ \\
& $\begin{array}{l}\text { Outboard sensor } \\
\text { Electronics box }\end{array}$ & $0.586 \mathrm{~kg}$ \\
& (EBOX) & $1.611 \mathrm{~kg}$ \\
& Total & $2.769 \mathrm{~kg}$ \\
& & $3.81 \mathrm{~W}$ \\
Power & Inboard sensor & $\pm 10,000 \mathrm{nT}$ \\
Dynamic range & Outboard sensor & $\pm 10,000 \mathrm{nT}$ \\
& & $1.19 \mathrm{pT}$ \\
Resolution & & $1 \mathrm{~Hz}, 32 \mathrm{~Hz}$, or $128 \mathrm{~Hz}$ \\
Sampling rate & & From DC to about $32 \mathrm{~Hz}$ \\
Frequency range & & Up to $10 \mathrm{pT} \mathrm{Hz}{ }^{-1 / 2}$ at $1 \mathrm{~Hz}$ \\
\hline Noise level & &
\end{tabular}

processes, depending on the scientific objectives of the mission (Balogh, 2010). The measured magnetic field vector differs from the true magnetic field due to the presence of various influencing factors. For MOMAG, the relationship of the calibrated field vector $\boldsymbol{B}_{C}$ to the measured field vector $\boldsymbol{B}_{M}$ can be written as follows:

$$
\boldsymbol{B}_{C}=\{\boldsymbol{\rho} \bullet \boldsymbol{\omega} \bullet \boldsymbol{\sigma}\}\left(\boldsymbol{B}_{M}-\boldsymbol{B}_{O}-\boldsymbol{B}_{S}\right) .
$$

Here, $\rho, \omega$, and $\boldsymbol{\sigma}$ are transformation matrix vectors of static rotation, orthogonality, and sensitivity, respectively. Variable $\rho$ can be derived from information on the spacecraft's attitude and orbit, along with the relative position of MOMAG sensors, whereas $\boldsymbol{\omega}$ and $\sigma$ can be determined during the ground calibration tests. Variable $\boldsymbol{B}_{O}$, the zero offset of the magnetometer, is always influenced

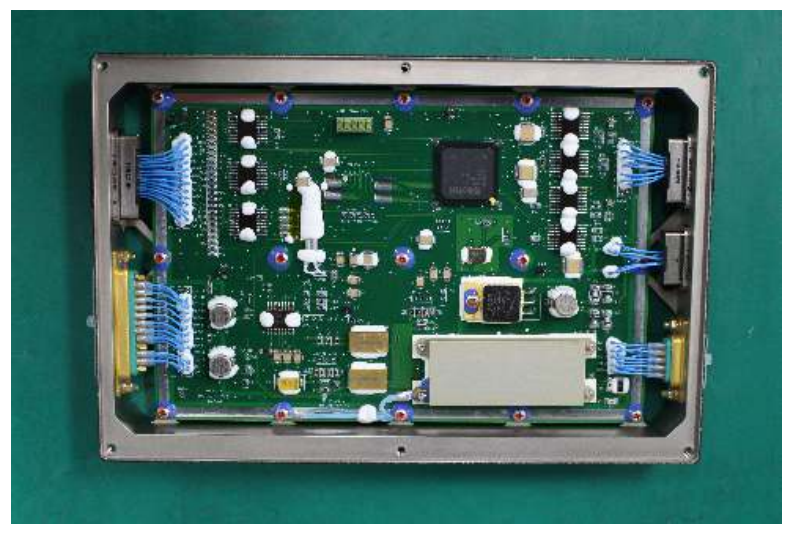

Figure 6. The instrument control unit (ICU).

by two factors, temperature and time. When the spacecraft is in orbit, the temperature evolves strongly. Thus, the temperature stabilities of the zero offsets need to be tested on the ground. Another factor that needs to be considered is that the sensor materials age over the duration of the mission, which will generate a long-term zero offset. Variable $\boldsymbol{B}_{S}$ is the spacecraft-generated field. The spacecraft is a complex system operating with hundreds of different models, leading to different field interferences. By using the dual-magnetometer configuration, $\boldsymbol{B}_{S}$ can be identified and eliminated from the in-flight data set.

\subsection{Ground Calibration}

MOMAG instrument underwent ground calibrations prior to the launch. The calibration flow was as follows:

(1) Calibration of the noise, offset, scale factor, and linearity were 
conducted in specialized facilities, such as the thermal chamber and shield container at the Magson laboratory in Berlin, Germany.

(2) Calibration of the sensor offset temperature stabilities (Magnes et al., 1998) in a low-field environment at temperatures from $-120^{\circ} \mathrm{C}$ up to $+25^{\circ} \mathrm{C}$. The calibration was conducted in a magnetic shielding-based facility at the Space Research Institute in Graz, Austria.

(3) Calibration of the offset, absolute gain, orthogonality, and transfer function in a coil-based facility at the Weak Magnetic Metering Station located in Yichang City, Hubei Province, China.

The following results obtained from MOMAG ground calibrations.

(1) The noise floor was found to be less than $7.8 \mathrm{pT} \mathrm{Hz}^{-1 / 2}$ for the IB sensor and $9.1 \mathrm{pT} \mathrm{Hz}^{-1 / 2}$ for the OB sensor (Figure 7).

(2) The gain drift was regarded as linear at a sufficient level and could be reproduced well.

(3) The sensor orthogonality was stable over the entire sensor temperature range.

(4) The offset drift was less than $1 \mathrm{nT}$ within a sensor temperature change of $100^{\circ} \mathrm{C}$.

\subsection{In-flight Calibration Plan}

MOMAG has been designed to contend with the effects of a magnetically unclean spacecraft. The spatial separation of the two magnetometers composing MOMAG is significantly smaller than the typical spatial scales of the natural magnetic field near Mars. Therefore, the difference in the measurements made by the two sensors is solely a function of the field generated by the spacecraft and the instrument offsets and noise. By applying the dualpoint measurement technique (Pope, 2008), which was developed for the magnetometer experiment on board the Venus Express (Zhang TL et al., 2006), we were able to identify most of the spacecraft interferences from the in-flight data set. The offset will be determined by using the nearly incompressible nature of Alfvén waves in the solar wind as a constraint (Hedgecock, 1975; Leinweber et al., 2008).

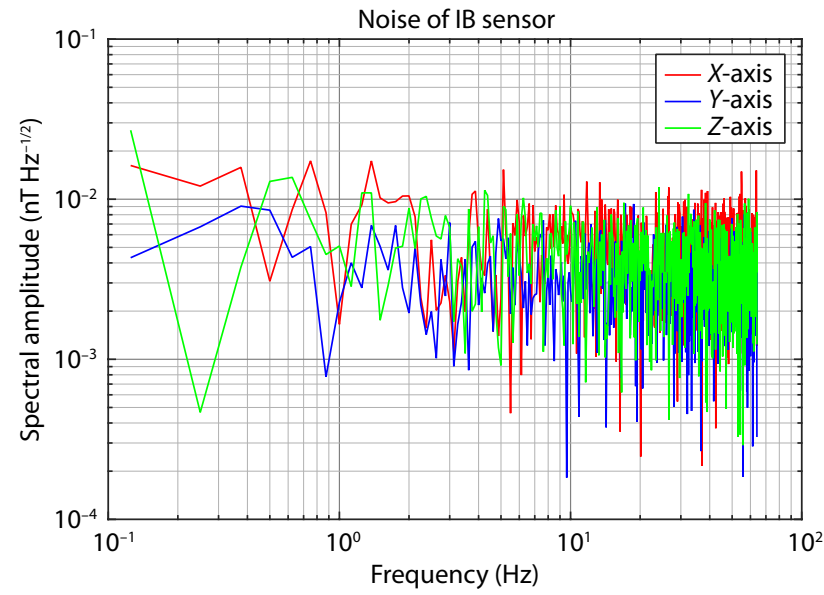

\section{Observation Plans}

After launch, the Tianwen-1 spacecraft will experience several phases, including an Earth-Mars transfer orbit phase, a Mars capture orbit phase, a Mars parking orbit phase, a Mars landing orbit phase, and a Mars satellite orbit phase. Before entering the Mars satellite orbit, MOMAG will turn on the power for self-inspection for several times. After releasing the lander with the rover, the orbiter will enter the Mars satellite orbit, which is an eclipse polar orbit (Figure 8). The boom will be unwound and locked up, forming a $45^{\circ}$ angle with the desk attached. MOMAG will then be powered on and will stay in operation status throughout the mission.

MOMAG uses different operational modes with different sampling rates to make the best use of the limited amount of telemetry for the scientific targets. MOMAG will be operated at a sampling frequency of $32 \mathrm{~Hz}$ when the orbiter is both near the periareon (about $265 \mathrm{~km}$ from the surface) and near the apoareon (about $11,945.6 \mathrm{~km}$ ) in time periods of $120 \mathrm{~min}$ and $60 \mathrm{~min}$, respectively. Throughout the rest of the orbit (about $288 \mathrm{~min}$ in total), MOMAG will be operated at a sampling frequency of $1 \mathrm{~Hz}$. The average data acquisition rate of MOMAG in a complete orbit is about 1.89 kilobits per second.

\section{Summary}

MOMAG will provide accurate vector magnetic field observations in the near-Mars space throughout the Mars satellite orbit phase of the mission at an alternating frequency between 32 and $1 \mathrm{~Hz}$. The instrumentation provides two-sensor vector measurements to eliminate the magnetic field generated by the spacecraft. MOMAG data, together with surface magnetic field vectors acquired by RoMAG and particle observations acquired by MINPA and MEPA, will open the door to comprehensive study of the magnetosphere and atmosphere of Mars.

\section{Acknowledgments}

The technical team of MOMAG is financially supported by the Chinese National Space Administration, and the science team of MOMAG is supported by the Strategic Priority Program (Grant No. XDB41000000) and the key Research Program of Frontier Sci-

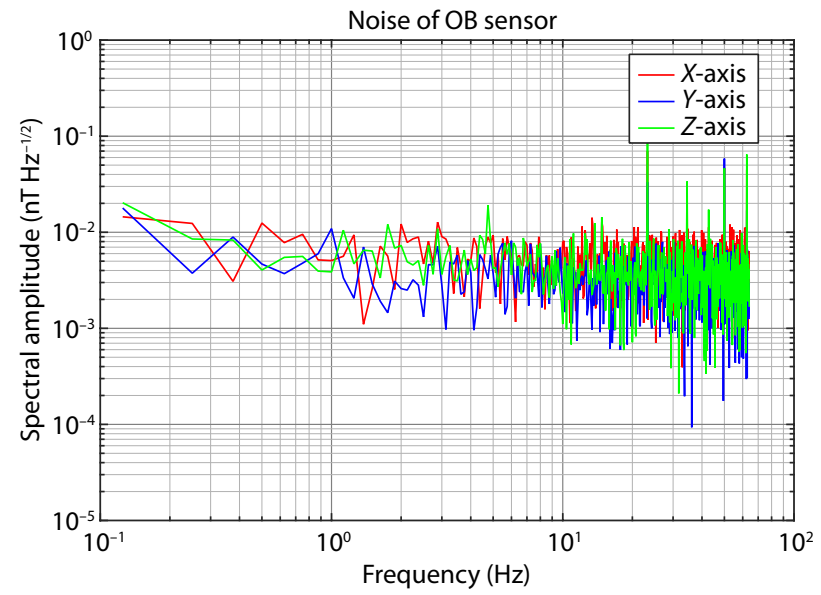

Figure 7. Noise profile of MOMAG sensor as a function of frequencies. IB, inboard; OB, outboard. 


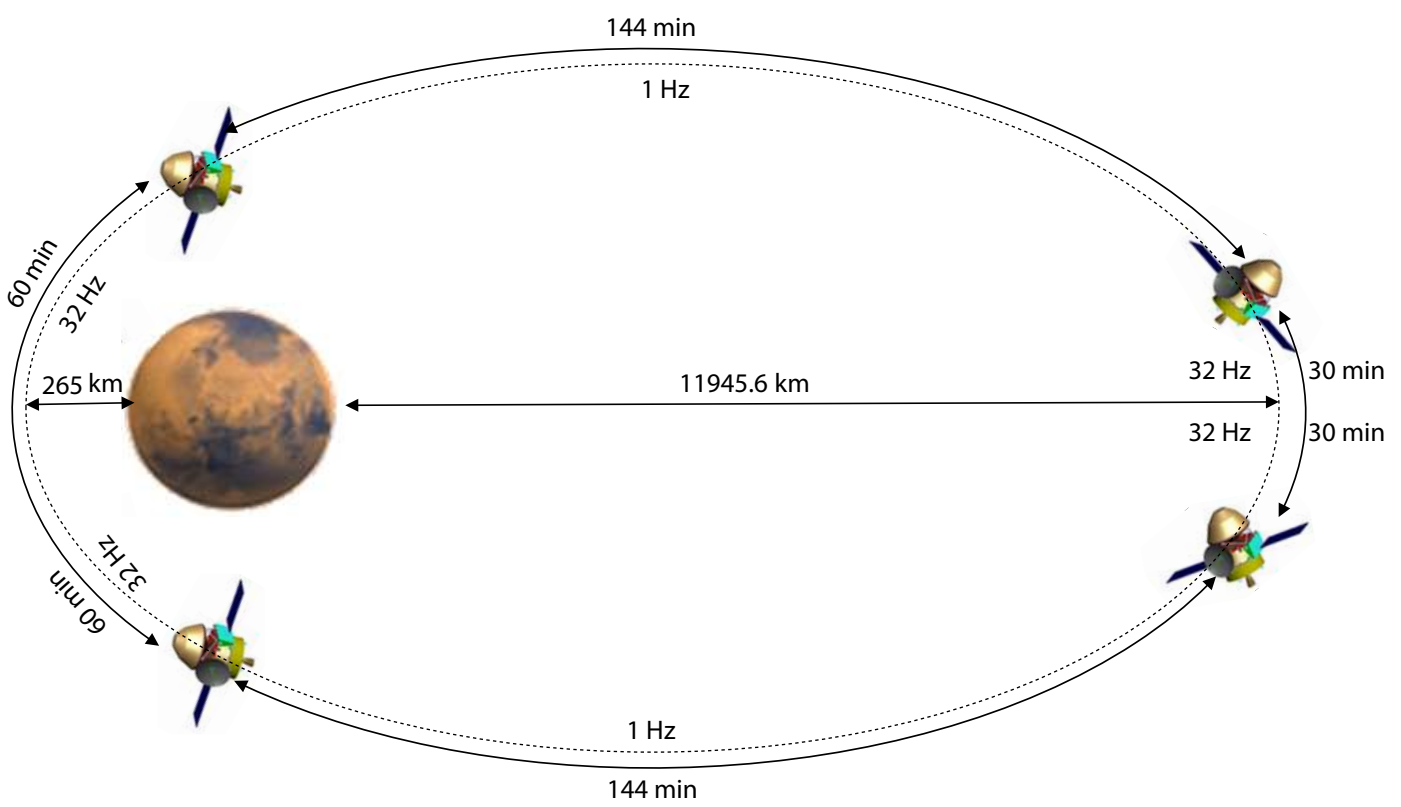

Figure 8. Observation plan of MOMAG in the Mars satellite orbit.

ences (No. QYZDB-SSW-DQC015) of the Chinese Academy of Sciences.

\section{References}

Acuña, M. H., Connerney, J. E. P., Wasilewski, P., Lin, R. P., Anderson, K. A., Carlson, C. W., McFadden, J., Curtis, D. W., Mitchell, D., ... Ness, N. F. (1998), Magnetic field and plasma observations at mars: initial results of the mars global surveyor mission. Science, 279(5357), 1676-1680. https://doi.org/10.1126/science.279.5357.1676

Auster, H. U., Apathy, l., Berghofer, G., Remizov, A., Roll, R., Fornacon, K. H., Glassmeier, K. H., Haerendel, G., Hejja, l., ... Waesch, R. (2007). ROMAP: Rosetta magnetometer and plasma monitor. Space Sci. Rev, 128, 221-240. https://doi.org/10.1007/s11214-006-9033-x

Auster, H. U., Glassmeier, K. H., Magnes, W., Aydogar, O., Baumjohann, W., Constantinescu, D., Fischer, D., Fornacon, K. H., Georgescu, E., ... Wiedemann, M. (2008). The THEMIS fluxgate magnetometer. Space Sci. Rev., 141(1-4), 235-264. https://doi.org/10.1007/s11214-008-9365-9

Balogh, A. (2010). Planetary magnetic field measurements: missions and instrumentation. Space Sci. Rev., 152(1-4), 23-97. https://doi.org/10.1007/s11214-010-9643-1

Bertucci, C., Mazelle, C., Crider, D.H., Vignes, D., Acuña, M.H., Mitchell, D.L., Lin, R.P. Connerney, J.E.P., Reme, H., Cloutier, P.A., Ness, N.F. and Winterhalter, D. (2003). Magnetic field draping enhancement at the Martian magnetic pileup boundary from Mars global surveyor observations. Geophysical Research Letters, 30(2), 1099.

Brain, D. A. (2006). Mars global surveyor measurements of the Martian solar wind interaction. Space Sci. Rev., 126(1-4), 77-112. https://doi.org/10.1007/s11214-006-9122-x

Connerney, J. E. P., Espley, J., Lawton, P., Murphy, S., Odom, J., Oliversen, R., and Sheppard, D. (2015). The MAVEN magnetic field investigation. Space Sci. Rev., 195(1-4), 257-291. https://doi.org/10.1007/s11214-015-0169-4

Dong, C. F., Bougher, S. W., Ma, Y. J., Toth, G., Nagy, A. F., and Najib, D. (2014). Solar wind interaction with Mars upper atmosphere: results from the oneway coupling between the multifluid MHD model and the MTGCM model. Geophys. Res. Lett., 41(8), 2708-2715. https://doi.org/10.1002/2014GL059515

Espley, J. R., Cloutier, P. A., Brain, D. A., Crider, D. H., and Acuña, M. H. (2004). Observations of low-frequency magnetic oscillations in the Martian magnetosheath, magnetic pileup region, and tail. J. Geophys. Res. Space Phys., 109(A7), A07213. https://doi.org/10.1029/2003JA010193
Georgescu, E., Auster, H. U., Takada, T., Gloag, J., Eichelberger, H., Fornaçon, K. H., Brown, P., Carr, C. M., and Zhang, T. L. (2008). Modified gradiometer technique applied to Double Star (TC-1). Adv. Space Res., 41(10), 1579-1584. https://doi.org/10.1016/j.asr.2008.01.014

Hedgecock, P. C. (1975). A correlation technique for magnetometer zero level determination. Space Sci. Instrum., 1(1), 83-90.

Leinweber, H. K., Russell, C. T., Torkar, K., Zhang, T. L., and Angelopoulos, V. (2008). An advanced approach to finding magnetometer zero levels in the interplanetary magnetic field. Meas. Sci. Technol., 19(5), 055104. https://doi.org/10.1088/0957-0233/19/5/055104

Ma, Y. J., Nagy, A. F., Sokolov, I. V., and Hansen, K. C. (2004). Three-dimensional, multispecies, high spatial resolution MHD studies of the solar wind interaction with Mars. J. Geophys. Res. Space Phys., 109(A7), A07211. https://doi.org/10.1029/2003JA010367

Magnes, W., Berghofer, G., Mocnik, K., Koren, W., Schwingenschuh, K., Stachel, M., Jernej, I., Riedler, W., Russell, C. T., ... Beek, T. J. (1998). A spaceborne magnetometer tested under extended temperature conditions (experiment MAREMF-OS/MARS-96). Meas. Sci. Technol., 9(8), 1219-1228. https://doi.org/10.1088/0957-0233/9/8/013

Müller, M., Lederer, T., Fornacon, K. H., and Schäfer, R. (1998). Grain structure, coercivity and high-frequency noise in soft magnetic Fe-81Ni-6Mo alloys. J. Magn. Magn. Mater., 177-181, 231-232. https://doi.org/10.1016/S03048853(97)00672-0

Ness, N. F., Behannon, K. W., Lepping, R. P., and Schatten, K. H. (1971). Use of two magnetometers for magnetic field measurements on a spacecraft. J. Geophys. Res., 76(16), 3564-3573. https://doi.org/10.1029/JA076i016p03564

Neubauer, F. M. (1975). Optimization of multimagnetometer systems on a spacecraft. J. Geophys. Res., 80(22), 3235-3240. https://doi.org/10.1029/JA080i022p03235

Pope, S. (2008). Methods for the detection and correction of magnetic noise in measurements made by magnetic field experiments onboard spacecraft [Ph. D. thesis]. Sheffield: University of Sheffield.

Primdahl, F. (1979). The fluxgate magnetometer. Journal of Physics E: Scientific Instruments, 12, 241-253. https://doi.org/10.1088/0022-3735/12/4/001

Zhang, T. L., Baumjohann, W., Delva, M., Auster, H. U., Balogh, A., Russell, C. T., Barabash, S., Balikhin, M., Berghofer, G., ... Lebreton, J. P. (2006). Magnetic field investigation of the Venus plasma environment: expected new results from Venus express. Planet. Space Sci., 54(13-14), 1336-1343. https://doi.org/10.1016/j.pss.2006.04.018 